\title{
The relationship of ovarian endometrioma and its size to the preoperative serum anti-Mullerian hormone level
}

\author{
Hyungjoon Yoon ${ }^{1 *} \mathbb{D}$, Hyunjoo Lee ${ }^{2 *}$, Seungchul Kim²(D), Jongkil Joo² \\ ${ }^{1}$ Pusan National University Graduate School, Busan, Republic of Korea \\ 2Pusan National University Hospital Medical Research Institute, Busan, Republic of Korea \\ *these authors contributed equally to this article
}

\begin{abstract}
Objectives: The aim of this study is to evaluate the impact of ovarian endometrioma according to its size on the serum anti-Mullerian hormone (AMH) levels compared to that of other benign ovarian cysts.

Material and methods: The current study retrospectively evaluated preoperative serum AMH level and its association to presenting ovarian cyst size which were measured in clinical setting. Women with surgically diagnosed endometrioma or other benign ovarian cysts were included. All patients underwent transvaginal or transrectal ultrasonography to determine the size of the ovarian cysts. Preoperative serum AMH level was checked and evaluated according to histologic type of the cyst, which were endometrioma or other benign ovarian cysts, respectively. Both groups were classified into $\leq 4 \mathrm{~cm},>4 \mathrm{~cm}$ and $\leq 8 \mathrm{~cm},>8 \mathrm{~cm}$ and $\leq 12 \mathrm{~cm},>12 \mathrm{~cm}$ according to the diameter of cyst and analyzed the difference of mean AMH levels in both groups.

Results: There was no significant difference in preoperative serum AMH level between the two groups ( $3.36 \pm 2.3$ versus $3.76 \pm 2.64, p=0.331)$. The difference of preoperative AMH levels according to categorized cyst size also was not statistically significant in both groups.

Conclusions: Preoperative serum AMH levels were not statistically different between endometrioma and other benign ovarian cyst groups and were not related to the size of endometrioma.
\end{abstract}

Key words: $\mathrm{AMH}$; endometriosis; endometrioma; ovarian cyst

Ginekologia Polska 2020; 91, 6: 313-319

\section{INTRODUCTION}

Anti-Müllerian hormone (AMH) is a dimeric glycoprotein that belongs to the transforming growth factor-beta family [1]. It is involved in the regression of the Müllerian ducts during male fetal development. In the female, AMH is solely produced by the granulosa cells of primary, preantral and small antral follicles in the ovaries [2].

Clinical aspects of AMH have some exclusive characteristics; the serum AMH level is closely related with age, with insignificant intra- and inter-cycle variation throughout menstrual cycles $[3,4]$. Because of such characteristics, $\mathrm{AMH}$ has been significantly used in several clinical practices such as assisted reproductive technologies $[5,6]$, prediction of menopause and diagnosis of PCOS [7-10]. Yet, while serum AMH level may be the best marker of ovarian reserve, the utility as a predictive marker for live births or timing of menopause is not reached to the definite conclusions [7].
In gynecological perspective with interests in clinical use of $\mathrm{AMH}$, the impact of a benign ovarian cyst on physiological serum AMH level is also one of the clinical issues with indefinite conclusion [11-13]. Such debate could be considered in two points; influence of surgery for an ovarian cyst and that of an ovarian cyst itself on the serum AMH level.

Regarding the influence of surgery on ovarian cysts and serum AMH level, it is widely accepted that ovarian reserve measured with serum AMH level would be reduced after ovarian cystectomy [14-16]. Henes et al. reported that serum AMH level was significantly decreased after surgery on a follicular cyst and endometriosis, but not on dermoid and other cysts [15].

When considering the influence of endometrioma on the serum AMH level, several subordinate concepts are needed to be pondered: the stage, laterality and size of endometrioma. Still, conflicting results are being observed; some reports have suggested that the serum AMH level is relatively low in 
the endometrioma group $[17,18]$, but the opposite findings have been also reported [19, 20]. Karadag et al. [17] reported lower $\mathrm{AMH}$ levels in patients with bilateral endometrioma than patients with unilateral endometrioma and lower $\mathrm{AMH}$ levels in women with deep infiltrating endometriosis. Similarly, Uncu et al. [18] found that endometrioma patients have lower AMH levels and antral follicle count when compared to the control and suggested that the presence of endometrioma itself is associated with a reduction in ovarian reserve. On the other hand, Kim et al. [19] suggested that the serum $\mathrm{AMH}$ level were not different between the endometrioma and teratoma groups, but in age-body mass index matched stage IV endometriosis, the serum AMH level was lower in the endometrioma group. Despite the ongoing debate, general consensus is that higher stage and bilaterality of endometrioma are more likely to result low AMH [21-23].

Expanding the issue to the relationship of size of endometrioma and serum AMH level, the controversies have been either insignificant or negative relationship of the two. However, the recent study of Marcellin et al. [20] has found strikingly positive association between the size of endometrioma and serum AMH level, introducing novel understanding of physiological characteristics of endometrioma and interpreting the associated AMH level. Currently, valuable scholarly investigations on $\mathrm{AMH}$ concentrations according to the size of endometrioma have been more complicated to be answered.

The aim of this study is to evaluate the impact of ovarian endometrioma according to its size on the serum anti-Mullerian hormone (AMH) levels compared to that of other benign ovarian cysts.

\section{MATERIAL AND METHODS}

\section{Patients}

This retrospective study was conducted at Department of Obstetrics and Gynecology, Pusan National University Hospital. Women with surgically diagnosed endometrioma between October 01, 2012 and October 01, 2019 were included as the study group. The exclusion criteria were prior ovarian surgery, irregular menstrual periods, the presence of polycystic ovary syndrome, hyperprolactinemia or abnormal thyroid function test (TFT), and medication history with dysmenorrhea management such as $\mathrm{GnRH}$ analogues, oral contraceptives or progestins during the past 3 months to the recruitment. The control group was comprised of women with benign ovarian cysts other than endometrioma. The same exclusion criteria were applied to the controls. BMI was calculated as the patient's weight in kilograms divided by her height in meters squared. All patients underwent laparoscopic or laparotomic ovarian cystectomy. All specimens obtained intraoperatively were submitted for pathologic examination, and pathologic confirmation was done in all cases. All patients agreed and provided informed consent forms indicating that their medical records were to be used for the study. The study was approved by the ethics committee of Pusan National University Hospital.

\section{Measurement of ovarian cyst size}

All patients underwent transvaginal or transrectal ultrasonography to determine the size of the endometriomas or other benign ovarian cysts using a 5-9 $\mathrm{MHz}$ transvaginal transducer or transrectally for virgin patients on the day before surgery (Voluson E6 General Electric, Milwaukee, Wauwatosa, WI, USA). The cyst size was recorded as the average of the largest and shortest diameters in centimeters. Each cyst diameters were measured vertically from outer-membrane to the opposite outer-membrane. Bilateral cysts were recorded as the sum of the two cyst sizes.

\section{Assay of AMH and inflammatory markers}

All of women underwent blood sampling for preoperative AMH measurements and inflammatory markers within a month prior to surgery. Blood samples were obtained from patients after $8 \mathrm{hrs}$ of overnight fast, including following measurements, using Roche Modular DP (Tokyo, Japan): complete blood cell count including number of white blood cells (WBC), percentages of segmented_neutrophils and lymphocytes and segmented neutrophil/lymphocyte (N/L) ratio. Serum AMH assay was performed using an anti-Mullerian hormone/Mullerian inhibiting substance Enzyme Immuno Assay (AMH/MIS EIA) kit (Immunotech version, Beckman Coulter, Marseille, France). The coefficients of variation of intra-assay and inter-assay were $12.3 \%$ and $14.2 \%$, respectively. The distribution of $\mathrm{AMH}$ values for two or three days of the physiological cycle according to the patient's age is determined by using $5 \%, 10 \%, 25 \%, 50 \%, 75 \%, 90 \%$, $95 \%$, and mean values according to age was calculated [24].

\section{Statistics}

All statistical data was organized into a computerized database. Variables were evaluated for clinical significance using chi-square test and Fisher's exact test for categorical variables or the independent $t$-test for continuous variables, where appropriate. One-way ANOVA was used to compare preoperative serum AMH levels according to categorized cyst sizes in the endometrioma group and control group. Pearson correlation coefficient was used for correlation between preoperative AMH level, age, ovarian cyst size and inflammatory markers. Using multiple linear regression analysis, factors affecting preoperative serum AMH level were studied. The statistical analysis was performed SPSS version 22 .

\section{RESULTS}

The mean age of the patients was higher in the endometrioma group compared to the control group, which 
were $30.45 \pm 5.99$ years and $28.09 \pm 7.55$ years, respectively $(p=0.034)$. Parity was lower in the endometrioma group than in the control group $(0.09 \pm 0.35$ versus $0.30 \pm 0.71$, $\mathrm{p}=0.033$ ) and showed a regular menstrual cycle compared to the control group ( $79.66 \%$ vs $20.34 \%, p<0.001$ ). In addition, dysmenorrhea in the endometrioma group were higher than the control group ( $82.2 \%$ vs $59.38 \%, p=0.001$ ). There were no significant differences in preoperative serum
AMH level between the two groups ( $3.36 \pm 2.3$ vs $3.76 \pm 2.64$, $p=0.331$ ). Serum WBC count and N/L ratio were significantly higher in endometriosis group than in control group (Tab. 1).

Table 2 shows the difference of preoperative $\mathrm{AMH}$ levels according to cyst size in endometrioma and control group. The groups were classified into $\leq 4 \mathrm{~cm},>4 \mathrm{~cm}$ and $\leq 8 \mathrm{~cm},>8 \mathrm{~cm}$ and $\leq 12 \mathrm{~cm},>12 \mathrm{~cm}$ according to the cyst size. The difference of preoperative AMH levels accord-

Table 1. Basic characteristics of the patients in control and endometrioma group

\begin{tabular}{|c|c|c|c|c|}
\hline & Overall $(n=182)$ & Control $(n=64)$ & Endometrioma $(n=118)$ & p value \\
\hline Age & $29.62(6.66)$ & $28.09(7.55)$ & 30.45 (5.99) & 0.034 \\
\hline BMI & $21.90(4.30)$ & $22.51(4.58)$ & $21.56(4.12)$ & 0.154 \\
\hline Smoking history* & & & & 1.000 \\
\hline Never & $175(96.15)$ & $62(96.88)$ & $113(95.76)$ & \\
\hline Current & $7(3.85)$ & $2(3.12)$ & $5(4.24)$ & \\
\hline Previous & $0(0.00)$ & $0(0.00)$ & $0(0.00)$ & \\
\hline Bilaterality* & & & & 0.310 \\
\hline No & $128(70.33)$ & $48(75)$ & $80(67.8)$ & \\
\hline Yes & $54(29.67)$ & $16(25)$ & $38(32.2)$ & \\
\hline Past history* & & & & 0.015 \\
\hline None & $168(92.31)$ & $59(92.19)$ & 109 (92.37) & \\
\hline HTN & $3(1.65)$ & $2(3.12)$ & $1(0.85)$ & \\
\hline $\mathrm{DM}$ & $1(0.55)$ & $0(0.00)$ & $1(0.85)$ & \\
\hline Hepatitis & $2(1.1)$ & $2(3.12)$ & $0(0.00)$ & \\
\hline Thyroid disease & $7(3.85)$ & $0(0.00)$ & $7(5.93)$ & \\
\hline Cancer & $1(0.55)$ & $1(1.56)$ & $0(0.00)$ & \\
\hline Gravidity & $0.37(0.86)$ & $0.52(1.04)$ & $0.30(0.74)$ & 0.139 \\
\hline Parity & $0.16(0.51)$ & $0.30(0.71)$ & $0.09(0.35)$ & 0.033 \\
\hline OCs* & & & & 1.000 \\
\hline Never & 175 (96.15) & 62 (96.88) & 113 (95.76) & \\
\hline Current & $4(2.2)$ & $1(1.56)$ & $3(2.54)$ & \\
\hline Previous & $3(1.65)$ & $1(1.56)$ & $2(1.69)$ & \\
\hline Menstruation* & & & & $<0.001$ \\
\hline Regular & $129(70.88)$ & $35(54.69)$ & $94(79.66)$ & \\
\hline Irregular & $53(29.12)$ & $29(45.31)$ & $24(20.34)$ & \\
\hline Dysmenorrhea* & & & & 0.001 \\
\hline No & $47(25.82)$ & $26(40.62)$ & $21(17.8)$ & \\
\hline Yes & 135 (74.18) & $38(59.38)$ & $97(82.2)$ & \\
\hline Menarche & $13.32(1.48)$ & $13.19(1.61)$ & $13.40(1.41)$ & 0.381 \\
\hline AMH (ng/mL) & $3.50(2.63)$ & $3.76(2.64)$ & $3.36(2.63)$ & 0.331 \\
\hline WBC (× 103/uL) & $6.66(2.03)$ & $6.23(1.56)$ & $6.90(2.22)$ & 0.019 \\
\hline Segmented_neutrophil (\%) & $59.35(10.65)$ & $58.07(9.93)$ & $60.05(11.01)$ & 0.218 \\
\hline Lymphocyte (\%) & $31.67(9.41)$ & $32.83(8.78)$ & $31.03(9.71)$ & 0.206 \\
\hline $\mathrm{N} / \mathrm{L}$ & $2.46(3.46)$ & $1.99(0.99)$ & $2.71(4.22)$ & 0.080 \\
\hline Cyst size $(\mathrm{cm})$ & $6.90(4.03)$ & $7.59(4.10)$ & $6.52(3.96)$ & 0.091 \\
\hline
\end{tabular}

Data are presented as the means (SD); ${ }^{*}$ values are presented as the number of patient (\%); $\mathrm{p}$ value by independent t-test for continuous variable; ${ }^{*} \mathrm{Chi}$-square test or Fisher's exact test for categorical variable; BMI — body mass index; $\mathrm{HTM}$ - hypertension; DM — diabetes mellitus; $\mathrm{OCs}$ - oral contraceptives; $\mathrm{AMH}$ - anti-

Mullerian hormone; WBC — white blood cell; $\mathrm{N} / \mathrm{L}$ — segmented neutrophil/lymphocyte ratio 
Table 2. Comparisons of serum AMH level according to cyst size in c' ontrol and endometrioma group

\begin{tabular}{|l|l|l|l|l|l|}
\hline Cyst size $(\mathbf{c m})$ & $\mathbf{4}$ & $\mathbf{4} \mathbf{4} \leq \mathbf{8}$ & $\mathbf{> 8 ~ \& \leq 1 2}$ & $\mathbf{> 1 2}$ & p value \\
\hline Overall $(\mathrm{n}=182)$ & $41,3.39(2.68)$ & $89,3.62(2.68)$ & $35,3.50(2.47)$ & $15,3.27(2.89)$ & 0.948 \\
\hline Control $(n=64)$ & $9,5.04(3.75)$ & $34,3.82(2.78)$ & $15,3.11(1.62)$ & $6,3.08(1.50)$ & 0.336 \\
\hline Endometrioma $(n=116)$ & $32,2.92(2.16)$ & $55,3.49(2.64)$ & $20,3.79(2.97)$ & $9,3.39(3.63)$ & 0.675 \\
\hline
\end{tabular}

A

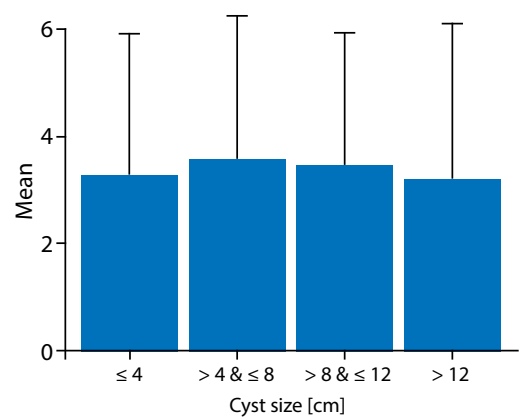

B
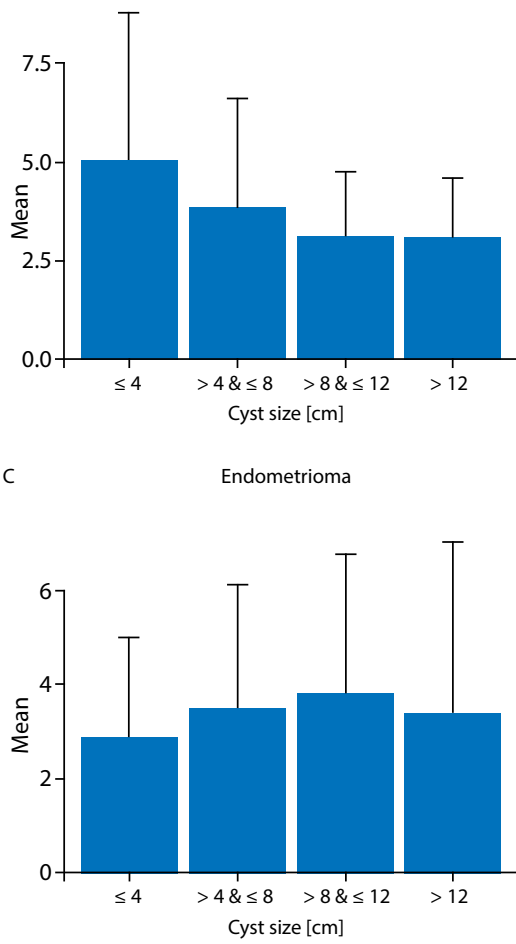

Figure 1. The value of the serum $\mathrm{AMH}$ according to average ovarian cyst size; A. Overall group; B. Control group including benign ovarian cyst except endometrioma; $C$. Endometrioma group

ing to categorized cyst size was not statistically significant in both groups ( $p=0.336, p=0.675$, respectively), regardless of bilaterality (Fig. 1).

Table 3 shows the results of the correlation between inflammatory markers and preoperative AMH levels. Pear-
Table 3. Correlations between preoperative AMH and other parameters including all groups

\begin{tabular}{|l|l|l|}
\hline ( $\mathbf{n = 1 8 2 )}$ & Rho value & $\mathbf{p}$ value \\
\hline Age [years] & -0.357 & $<0.0001$ \\
\hline Average of cyst size $[\mathrm{cm}]$ & -0.051 & 0.492 \\
\hline N/L ratio & -0.022 & 0.766 \\
\hline Number of WBC & -0.009 & 0.907 \\
\hline
\end{tabular}

AMH — anti-Mullerian hormone; N/L — segmented neutrophil/lymphocyte; WBC — white blood cell

son correlation coefficient showed only age factors have a significantly negative correlation (Rho value $=-0.357$ with $p<0.0001)$. Serum WBC count and N/L ratio had Rho values of $-0.009(p=0.907)$ and $-0.022(p=0.766)$, respectively.

The result of multiple linear regression analysis including age, cyst size, serum WBC count and N/L ratio showed non-significant statistical correlation except age (coefficient $\beta=-0.15, p<0.0001)$.

\section{DISCUSSION}

The surgery-related decrease of ovarian reserve in endometriosis is relatively well-established finding $[2,15,16,18]$. However, the impact of ovarian endometrioma itself on ovarian reserve - one of the most important preoperative characteristics to decide further treatment method - showed inconsistent results. Uncu et al. [18] reported that women with endometrioma had lower $\mathrm{AMH}$ levels and antral follicle count than controls, and Pacchiarotti et al. [25] also observed the negative effect of endometriosis on the ovarian reserve, especially in cases of severe endometriosis. The possible underlying mechanism suggested by the authors was that increased peritoneal macrophages in endometriosis could have caused substantial damage to ovarian tissue and oxidative stress, inducing oocyte degeneration and apoptosis by disturbing the meiotic spindle [25, 26]. Yet, in these studies, the control groups comprised women of reproductive age who did not have any ovarian cysts [18]. Thus, it would be unclear to conclude whether such reduced serum AMH level in the endometrioma group was caused specifically by endometrioma or generally by the presence of cystic lesion on the ovary. When the control group had benign ovarian cysts, some studies have reported the insig- 
nificant difference in AMH level between the control and the patient group with endometrioma [12, 19]. More detailed evaluation and analysis according to the characteristics of endometrioma itself and suitable control group are required to interpret $\mathrm{AMH}$ level in endometrioma patients.

Recently, Marcellin et al. [20] evaluated serum AMH levels in endometrioma patients according to the size of the cyst; interestingly, they reported that serum AMH levels in women with no prior history of surgery for endometrioma increased with the cyst size of endometrioma. In the control group, non-endometrioma benign ovarian cyst group, serum AMH levels was not related with the size of cyst. The authors concluded that serum AMH level had positive correlation with endometrioma size and was not affected by laterality of endometrioma or deep infiltrating endometriosis, which was strikingly different from previous literature.

In this study, to investigate such inconclusive effect of endometrioma itself on serum AMH level according to its size, we compared the preoperative AMH level between endometrioma and other benign ovarian cyst group, conducting subgroup analysis according to corresponding ovarian cyst size.

First, we observed that the effect of benign ovarian cyst on serum AMH level was not different between the endometrioma and the other benign ovarian cyst group. Such result of ours was in agreement with the study of Streuli l et al. [13], in which they reported that the serum AMH levels was not different between women with endometriosis and women with benign gynecological condition (endometrioma group, $3.6 \pm 3.1 \mathrm{ng} / \mathrm{mL}$ and control group, $4.1 \pm 3.4 \mathrm{ng} / \mathrm{mL}, \mathrm{p}=0.06$ ). Therefore, preoperative serum AMH level seems to be similar between endometrioma and other benign ovarian cysts, as previously known $[13,20]$.

Next, we compared the relationship of ovarian cyst size and serum AMH level. In our study, there was no statistical difference between endometrioma size and serum AMH level, which was inconsistent with the results of Marcellin et al. However, except for the endometrioma with size of more than $12 \mathrm{~cm}$, the serum AMH level tended to be increased with the increasing cyst size. Additionally, we have run the same statistical analysis on unilateral endometrioma and bilateral endometrioma separately, and, interesting enough, the similar pattern was observed (data not shown). Despite statistical insignificance, in unilateral endometrioma, serum $\mathrm{AMH}$ level tended to be increased with increasing cyst size up to $6 \mathrm{~cm}$ and dropped thereafter; in bilateral endometrioma, the same tendency was observed up to the cyst size of $12 \mathrm{~cm}$. At this point, we supposed that serum AMH level increased with increasing endometrioma size up to a certain limit - in this study, the upper limit was $12 \mathrm{~cm}$ in diameter when the unilateral and bilateral endometrioma were combined - and became insignificant thereafter.
Though further statistical analysis on subdivided sizes of endometrioma is warranted, based on the current results, the size of endometrioma might have affected the serum AMH level, not necessarily reflecting the ovarian reserve, which concurs with the results of Marcellin et al. [20].

Marcellin et al. suggested three hypotheses to explain their results [20]. First is selection bias. In case of women with low AMH levels, surgical management tended to be avoided. Second, they suggested that there may be increased secretion of AMH into the circulation by the ovaries as the size of the endometrioma increases; inflammation and neoangiogenesis in endometriosis could boost the local blood clearance from the ovaries. Third, the toxicity of endometrioma on the ovarian reserve may contribute improved primordial follicular stimulation and consequently an increase in serum AMH levels.

Of the three hypotheses of Marcellin et al. [20], regarding the selection bias, we also excluded infertile patients, suggesting the potential of selection bias may exist. Streuli et al. [13] excluded infertile patients who were more likely to have lower basal AMH in their study as well, and they reported similar results to ours. Considering the effect of infertile patient, Kim et al. [19] reported that the preoperative serum $\mathrm{AMH}$ was not different between endometrioma and mature cystic teratoma, including infertile patients in their subject group (endometrioma group 9.8\% and control group 7.8\%, $\mathrm{p}=$ not significant, respectively); additionally, in case of stage IV endometriosis, the serum AMH level was lower in the endometrioma group. Thus, regardless of including infertile patient to the subject group, the basal serum $\mathrm{AMH}$ level was not significantly different between patients with endometrioma or other benign ovarian cysts. Still, more detailed and larger studies are needed considering infertility history and endometrioma severity.

For evaluating the effect of inflammation, we analyzed the relationship of inflammation markers; WBC count and $\mathrm{N} / \mathrm{L}$ ratio. The results showed no statistical relevance. However, it is hard to define that the number of serum WBCs and $\mathrm{N} / \mathrm{L}$ ratio reflected the inflammatory environment of peritoneal cavity related with endometrioma. It is necessary to analyze the inflammatory marker of peritoneal fluid.

Last, as reported elsewhere, an increase in endometrioma size might cause increasing toxicity on the ovarian reserve, and short-term exposure of human ovarian follicles to cyclophosphamide metabolites seems to promote follicular activation in vitro $[27,28]$. However, such studies were conducted in vitro, and the toxicity of cyclophosphamide metabolite and endometrioma might differ in clinical setting.

The current study has several limitations. First, the number of patients included in this study was relatively small. However, data were collected in a single institute, and all patients were firmly diagnosed by laparoscopic surgery. 
We clearly excluded minimal or mild endometriosis patients in control group by laparoscopy, leading the consistency of data to be quite valuable. Second, the mean age of control group was significantly lower than the endometrioma group. Despite the slightly higher mean age, the mean $\mathrm{AMH}$ of the endometrioma group was comparable to the control group. Thus, we could logically conclude that the preoperative AMH were not different between the endometrioma and control groups. Lastly, since the current study is retrospective, all statistical analysis was inevitably performed using previously measured data; yet, Marcellin et al. [20] adopted the sum of the largest diameters for bilateral ovarian cysts in their study of increasing serum $\mathrm{AMH}$ level with endometrioma size, when, in previous literature, women with bilateral ovarian cysts had been reported to have lower serum AMH levels, irrespective of the nature of the cyst [12]. In the current study, regardless of bilaterality of ovarian cysts, increasing tendency of serum AMH level with ovarian cyst size was observed, giving the important perspective of relationship between serum AMH level and the size of cyst in endometrioma.

\section{CONCLUSIONS}

The preoperative serum AMH levels were not statistically different between the endometrioma and other benign ovarian cyst groups and were not related to the size of endometrioma. Nevertheless, except for the cysts larger than $12 \mathrm{~cm}$ in diameter, the serum AMH level tended to have positive relationship with increasing endometrioma size. With further analyses with larger number of patients and more suitable inflammatory markers, future findings on the association between serum AMH level and endometrioma size could innovatively suggest proper interpretation of preoperative serum AMH level and clear management direction for treatment in endometrioma patients.

\section{Conflict of interest}

None of the authors have direct or indirect conflict interest associated with publishing the article.

\section{REFERENCES}

1. Li HW, Ng EH, Wong BP, et al. Correlation between three assay systems for anti-Müllerian hormone (AMH) determination. J Assist Reprod Genet. 2012; 29(12): 1443-1446, doi: 10.1007/s10815-012-9880-1, indexed in Pubmed: 23117477.

2. Anderson RA, Nelson SM, Wallace WHB. Measuring anti-Müllerian hormone for the assessment of ovarian reserve: when and for whom is it indicated? Maturitas. 2012; 71(1): 28-33, doi: 10.1016/j.maturitas.2011.11.008, indexed in Pubmed: 22119275.

3. La Marca A, Stabile G, Artenisio AC, et al. Serum anti-Mullerian hormone throughout the human menstrual cycle. Hum Reprod. 2006; 21(12): 3103-3107, doi: 10.1093/humrep/del291, indexed in Pubmed: 16923748.

4. Streuli I, Fraisse T, Pillet C, et al. Serum antimüllerian hormone levels remain stable throughout the menstrual cycle and after oral or vaginal administration of synthetic sex steroids. Fertil Steril. 2008; 90(2): 395-400, doi: 10.1016/j.fertnstert.2007.06.023, indexed in Pubmed: 17919608.
5. Nardo LG, Gelbaya TA, Wilkinson H, et al. Circulating basal anti-Müllerian hormone levels as predictor of ovarian response in women undergoing ovarian stimulation for in vitro fertilization. Fertil Steril. 2009; 92(5): 1586-1593, doi: 10.1016/j.fertnstert.2008.08.127, indexed in Pubmed: 18930213.

6. La Marca A, Papaleo E, Grisendi V, et al. Development of a nomogram based on markers of ovarian reserve for the individualisation of the follicle-stimulating hormone starting dose in in vitro fertilisation cycles. BJOG. 2012; 119(10): 1171-1179, doi: 10.1111/j.1471-0528.2012.0 3412.x, indexed in Pubmed: 22805536.

7. Iwase A, Nakamura T, Osuka S, et al. Anti-Müllerian hormone as a marker of ovarian reserve: What have we learned, and what should we know? Reprod Med Biol. 2016; 15(3): 127-136, doi: 10.1007/s12522-015-0227-3, indexed in Pubmed: 29259429.

8. Song DoK, Oh JY, Lee H, et al. Differentiation between polycystic ovary syndrome and polycystic ovarian morphology by means of an anti-Müllerian hormone cutoff value. Korean J Intern Med. 2017; 32(4): 690-698, doi: 10.3904/kjim.2016.038, indexed in Pubmed: 27899014.

9. Iliodromiti S, Kelsey TW, Anderson RA, et al. Can anti-Mullerian hormone predict the diagnosis of polycystic ovary syndrome? A systematic review and meta-analysis of extracted data. J Clin Endocrinol Metab. 2013; 98(8): 3332-3340, doi: 10.1210/jc.2013-1393, indexed in Pubmed: 23775353.

10. Broer SL, Eijkemans MJC, Scheffer GJ, et al. Anti-mullerian hormone predicts menopause: a long-term follow-up study in normoovulatory women. J Clin Endocrinol Metab. 2011; 96(8): 2532-2539, doi: 10.1210/jc.2010-2776, indexed in Pubmed: 21613357.

11. Kitajima M, Khan KN, Harada A, et al. Association between ovarian endometrioma and ovarian reserve. Front Biosci (Elite Ed). 2018; 10: 92-102, doi: 10.2741/e810, indexed in Pubmed: 28930606.

12. Somigliana E, Marchese MA, Frattaruolo MP, et al. Serum anti-mullerian hormone in reproductive aged women with benign ovarian cysts. Eur J Obstet Gynecol Reprod Biol. 2014; 180: 142-147, doi: 10.1016/j. ejogrb.2014.06.009, indexed in Pubmed: 25009087.

13. Streuli I, de Ziegler D, Gayet V, et al. In women with endometriosis anti-Müllerian hormone levels are decreased only in those with previous endometrioma surgery. Hum Reprod. 2012; 27(11):3294-3303, doi: 10.1093/humrep/des274, indexed in Pubmed: 22821432.

14. Chang HJ, Han SH, Lee JR, et al. Impact of laparoscopic cystectomy on ovarian reserve: serial changes of serum anti-Müllerian hormone levels. Fertil Steril. 2010; 94(1): 343-349, doi: 10.1016/j.fertnstert.2009.02.022, indexed in Pubmed: 19345350.

15. Henes M, Engler T, Taran FA, et al. Ovarian cyst removal influences ovarian reserve dependent on histology, size and type of operation. Womens Health (Lond). 2018; 14: 1745506518778992, doi: 10.1177/1745506518778992, indexed in Pubmed: 29806554.

16. Kwon SuK, Kim SH, Yun SC, et al. Decline of serum antimüllerian hormone levels after laparoscopic ovarian cystectomy in endometrioma and other benign cysts: a prospective cohort study. Fertil Steril. 2014; 101(2): 435-441, doi: 10.1016/j.fertnstert.2013.10.043, indexed in Pubmed: 24290000.

17. Karadağ C, Yoldemir $T$, Karadağ SD, et al. The effects of endometrioma size and bilaterality on ovarian reserve. J Obstet Gynaecol. 2019; 40(4): 531-536, doi: 10.1080/01443615.2019.1633518.

18. Uncu G, Kasapoglu I, Ozerkan K, et al. Prospective assessment of the impact of endometriomas and their removal on ovarian reserve and determinants of the rate of decline in ovarian reserve. Hum Reprod. 2013; 28(8): 2140-2145, doi: 10.1093/humrep/det123, indexed in Pubmed: 23624580.

19. Kim JuY, Jee BC, Suh CS, et al. Preoperative serum anti-mullerian hormone level in women with ovarian endometrioma and mature cystic teratoma. Yonsei Med J. 2013; 54(4): 921-926, doi: 10.3349/ymj.2013.54.4.921, indexed in Pubmed: 23709427.

20. Marcellin L Santulli $P$, Bourdon $M$ et al Serum antimüllerian hormone concentration increases with ovarian endometrioma size. Fertil Steril. 2019; 111(5): 944-952.e1, doi: 10.1016/j.fertnstert.2019.01.013, indexed in Pubmed: 30878253

21. Goodman LR, Goldberg JM, Flyckt RL, et al. Effect of surgery on ovarian reserve in women with endometriomas, endometriosis and controls. Am J Obstet Gynecol. 2016; 215(5): 589.e1-589.e6, doi: 10.1016/j. ajog.2016.05.029, indexed in Pubmed: 27242204.

22. Leone Roberti Maggiore U, Scala C, Venturini PL, et al. Endometriotic ovarian cysts do not negatively affect the rate of spontaneous ovulation. Hum Reprod. 2015; 30(2): 299-307, doi: 10.1093/humrep/deu308, indexed in Pubmed: 25432923. 
23. Maggiore UL, Gupta J, Ferrero S. Treatment of endometrioma for improving fertility. Eur J Obstet Gynecol Reprod Biol. 2017; 209: 81-85, doi: 10.1016/j.ejogrb.2016.02.035.

24. Lee J, Park D, Kim ML, et al. Age-related distribution of anti-Müllerian hormone levels in 2,879 Korean women with regular menstruation. Korean Journal of Obstetrics \& Gynecology. 2012; 55(12): 920, doi: 10.5468/kjog.2012.55.12.920.

25. Pacchiarotti A, Frati P, Milazzo GN, et al. Evaluation of serum anti-Mullerian hormone levels to assess the ovarian reserve in women with severe endometriosis. Eur J Obstet Gynecol Reprod Biol. 2014; 172: 62-64, doi: 10.1016/j.ejogrb.2013.10.003, indexed in Pubmed: 24210790 .
26. Halis $G$, Arici A. Endometriosis and inflammation in infertility. Ann N Y Acad Sci. 2004; 1034: 300-315, doi: 10.1196/annals.1335.032, indexed in Pubmed: 15731321

27. Lande Y, Fisch B, Tsur A, et al. Short-term exposure of human ovarian follicles to cyclophosphamide metabolites seems to promote follicular activation in vitro. Reprod Biomed Online. 2017; 34(1): 104-114, doi: 10.1016/j.rbmo.2016.10.005, indexed in Pubmed: 27815062.

28. Sanchez AM, Viganò $P$, Somigliana $E$, et al. The distinguishing cellular and molecular features of the endometriotic ovarian cyst: from pathophysiology to the potential endometrioma-mediated damage to the ovary. Hum Reprod Update. 2014; 20(2): 217-230, doi: 10.1093/humupd/dmt053, indexed in Pubmed: 24129684. 